\title{
Multicriteria optimization of interdependent project portfolios with 'a priori' incorporation of decision maker preferences
}

\author{
Laura Cruz ${ }^{1}$, Eduardo R. Fernandez ${ }^{2}$, Claudia G. Gomez ${ }^{1}$ and Gilberto Rivera ${ }^{1}$ \\ ${ }^{1}$ Madero Institute of Technology, Cd. Madero, Mexico \\ ${ }^{2}$ Autonomous University of Sinaloa, Culiacán, Mexico \\ lauracruzreyes@itcm.edu.mx, eddyf@uas.edu.mx,cggs71@hotmail.com, riveragil@gmail.com
}

\begin{abstract}
One of the most important management issues lies in determining the best portfolio of a given set of investment proposals. This decision involves the pursuit of multiple criteria, and has been commonly addressed by implementing a two-phase procedure whose first step identifies the efficient solution space. In this paper we introduce our algorithm called Non-Outranked Ant Colony Optimization (NO-ACO) that optimizes portfolios with interprojects interactions whilst takes into account the DM's preferences by incorporating a priori preferences articulation. Experimental tests show the advantages of our proposal over the two-phase approach. Also, NO-ACO performed particularly well for problems with high dimensionality.
\end{abstract}

Keywords: portfolio selection, interdependent projects, multicriteria optimization, preferences incorporation.

\section{Introduction}

Portfolio problems are ubiquitous in business and government organizations. Usually, there are more good ideas for projects or programmes than resources (funds, capacity, time, etc.) to support them ([1]). Manufacturing enterprises recognize that success depends on the selection of research and development (R\&D) project portfolios, expecting that these projects permit them to develop new products that generate growing benefits. Local governments allocate public funds to projects and programmes that improve social and educational service. Environmental regulations and alternative policy measures attempt to mitigate harmful consequences of human activity ([2]). To fight poverty, governments in underdeveloped countries fund many helpful social programmes.

Portfolio consequences are usually described by multiple attributes related to the organizational strategy. A vector $z(x)=\left\langle z_{1}(x), z_{2}(x), \ldots, z_{p}(x)\right\rangle$ is associated to the consequences of a portfolio $x$ considering $p$ criteria. This is a vector representation of the portfolio's impact. In the simplest case, $z(x)$ is obtained from a cumulative sum of benefits of the selected projects, but under interacting pro- ject conditions, it is necessary to consider the contribution of interdependent project groups. Without loss of generality, we can assume that higher criterion values are preferred to lower values. The best portfolio is obtained by solving:

$$
\max _{x \in R_{F}}\left\{<z_{1}(x), z_{2}(x), \ldots, z_{p}(x)>\right\}
$$

where $R_{F}$ is the feasible portfolios space, usually determined by the available budget, and by constraints for the kinds of projects, social roles and geographic zones. Problem 1 is badly defined mathematically, yet people must solve it. To solve Problem 1 means to find the best compromise solution according to the system of preferences and values of the decision maker (DM).

In the scientific literature, the problem expressed by (1) has received great interest in $R \& D$ management by manufacturing and industrial enterprises (e.g. [3, 4, 5, 6, $7,8]$ ). Most of these approaches can also be applied in public sector. Perhaps, what best characterizes the portfolio problems in non-profit organizations are the emphasis on intangible criteria and, likely, a higher number of project proposals and objectives to optimize. For example, in socially responsible organizations, the number of criteria used for capital investment may be about a dozen ([9]). Even more objective functions should be considered in basic research project management (cf. [10]). A high number of project proposals can apply for public support in a simple call for projects. For instance, in 2012 the U. S. state of Georgia had a list of over 1600 applicant projects only at the State Department of Transportation ([11, $12,13,14])$. There should be a large set of Paretoefficient solutions to Problem 1. However, the decision maker has to select only one portfolio according to her/his preferences on the portfolio's consequences expressed by $z(x)$.

\section{An outline of the state of the art}

Only non-dominated solutions to (1) can fulfill the necessary conditions for being considered the best portfolio. So, most solution methods seek to generate the Pareto frontier, and later, by some interactive method, multicriteria procedure or heuristic, try to identify the best 
compromise. These approaches assume that the DM has the capacity to make valid judgments about the set of efficient points until reaching the best compromise. This way to identify the best solution is commonly referred as the a posteriori preferences modeling (cf. [15]).

Ghasemzadeh et al. ([16]) model preferences using a weighted-sum function. They approximate the Pareto frontier by changing the weights and solving the resultant model by $0-1$ programming. Stummer and Heidenberger in [5] include synergy and redundancy in selecting R\&D projects; their procedure consists of three phases: 1) filtering the proposals and retaining those most promissory projects in order to reduce the set of projects to a "manageable" size, 2) generating the efficient frontier of portfolios for the reduced set by an integer linear programming method, and 3) supporting the decision making process, helping the DM to identify the best compromise by an interactive process.

However, most recent works show the advantages of multiobjective metaheuristics methods to approximate the Pareto set (e.g. [8, 17, 18, 19, 20, 21, 22, 23]). In [24] Doerner et al. combine Ant Colony Optimization (ACO) with 0-1 dynamic mathematical programming to initialize the algorithm with enhanced solutions. One of the most complete proposals was suggested by Carazo et al. in [18, 25], which model interactions among projects (such as Stummer and Heidemberger in [5]) and temporal dependencies, enabling the allocation of resources not used in previous periods. By means of a Scatter Search, Carazo et al. ([18]) outperform SPEA2 [26] in the range of 25-60 projects considering up to six objective functions.

Compared to multi-objective optimization methods based on mathematical programming, metaheuristic approaches exhibit relevant advantages: 1) they have the ability to deal with a set of solutions (called population) at the same time, allowing to approximate the efficient frontier in a single algorithm run, and 2) they are less sensitive to the mathematical properties of objective functions and problem constraints ([27]).

Despite their advantages, most metaheuristic algorithms are degraded when trying to solve problems with more than a small number of objectives ([28, 29]). Also, when they try to approximate the efficient frontier, generate a very large amount of solutions. This exceeds the cognitive abilities of an average DM to identify satisfactorily the best compromise. Even if we could apply the multicriteria decision analysis methods, this process can turn too hard, because these methods do not perform well on decision problems with so many alternatives.

In order to address these drawbacks, in [30] Fernandez et al. proposed a method of preference incorporation in multiobjective evolutionary optimization, which was after extended in [10] to project portfolio optimization. They use a fuzzy outranking preference system to identify a small privileged subset of Pareto-efficient solutions. The model is independent of the number of criteria considered by the DM, and achieves to solve instances in a range of $100-500$ projects and 9-16 objectives. Another advantage is its high tolerance to imprecise objective values, and its capacity of handling ordinal and qualitative criteria. However, the model of Fernandez et al. ([10, 30]) does not consider interactions among projects, what is an important concern in most practical applications.

In light of this feedback, we propose a portfolio optimization metaheuristic approach based on the preferential model of Fernandez et al. ([10]). So, our metaheuristic inherits all advantages of their model, but we have incorporated the capacity to solve portfolios with interdependent projects. Several papers in the literature consider the synergy as an inherent characteristic of the portfolio problem (e.g. [5, 18, 24, 31]). Our solution approach, called Non-Outranked Ant Colony Optimization (NO-ACO) shows promising results compared to other related algorithms. Experimental results provide evidence that is very capable to get close to the Pareto frontier when is looking for the best compromise.

\section{Preference incorporation in multicriteria optimiza- tion metaheuristic approaches}

Because it would be difficult to determine the Pareto frontier in real applications, most search algorithms are limited to a predetermined number of efficient solutions. With the intention of finding a representative sample of the Pareto frontier, some algorithms include distance measures that favor the spread among solutions (e.g. [32, 33]). However, this do not ensure that the best compromise can be found, and if even so, the solution set exceeds the capacity of an average DM to make the decision process successfully.

In order to make easier the decision making phase, the DM would agree with incorporating his/her multicriteria preferences into the search process. This preference information is used to guide the search towards the Region Of Interest (ROI) ([34]), the privileged zone of the Pareto frontier that best matches the DM's preferences.

DM preference information can be expressed in different ways. According to Bechikh ([35]), the most commonly used ways are the following:

- those in which importance factors (weights) are assigned by the DM to each objective function (e.g. [36, 37]),

- those in which the DM makes pair-wise comparisons on a subset of the current population in order to rank the sample's solutions (e.g. [38, 39]),

- when pair-wise comparisons between pairs of objective functions are performed in order to rank the set of objective functions (e.g. [40, 41]),

- those based on goals or aspiration levels to be achieved by each objective (reference point) (e.g. [42, 43]),

- when the DM identifies acceptable trade-offs between objective functions (e.g. [44]);

- when the DM supplies the model's parameters to build a fuzzy outranking relation (e.g. [10, 30]); 
- construction of a desirability function which is based on the assignment of some desirability thresholds (e.g. [45]).

In the field of portfolio optimization, the model of Fernandez et al. ([10]) has shown substantial benefits for tackling these problems. This model is briefly explained below.

\subsection{The best portfolio in the sense of Fernandez et al. ([10])}

The proposal by Fernandez et al. ([10, 30]) is based on the relational system of preferences described by Roy in [46]. A crucial model is the degree of credibility of the statement " $x$ is at least as good as $y "$; this is represented as $\sigma(x, y)$ and could be calculated using proven methods of literature, such as ELECTRE ([47]) and PROMETHEE ([48]). The proposal by Fernandez et al. ([10]) identifies one of the following relations for each pair of portfolios $(x, y)$ controlled by the parameters $\lambda, \beta$, and $\varepsilon(0 \leq \varepsilon \leq \beta \leq$ $\lambda$ and $\lambda \geq 0.5)$ :

1) Indifference: From the DM perspective, both alternatives have a high degree of equivalence; therefore he/she cannot state that one is preferred over other. This relationship is denoted as $x \mathrm{I} y$. In terms of $\sigma(x, y)$ is defined as the conjunction of:

$$
\begin{array}{ll}
\text { a. } & \sigma(x, y) \geq \lambda \wedge \sigma(y, x) \geq \lambda \\
\text { b. } & |\sigma(x, y)-\sigma(y, x)| \leq \varepsilon .
\end{array}
$$

2) Strict preference: Denoted as $x \mathrm{P} y$, represents the situation when the DM significantly prefers $x$. It is defined as a disjunction of the conditions:

$$
\begin{array}{ll}
\text { a. } & x \text { dominates } y . \\
\text { b. } & \sigma(x, y) \geq \lambda \wedge \sigma(y, x)<0.5 . \\
\text { c. } & \sigma(x, y) \geq \lambda \wedge(0.5 \leq \sigma(y, x) \leq \lambda) \wedge(\sigma(x, y)- \\
& \sigma(y, x)) \geq \beta .
\end{array}
$$

3) Weak preference: Represented as $x \mathrm{Q} y$, models a state of doubt between $x \mathrm{P} y$ and $x \mathrm{I} y$. It can be defined as the conjunction of:

$$
\begin{array}{ll}
\text { a. } & \sigma(x, y) \geq \lambda \wedge \sigma(x, y) \geq \sigma(y, x) . \\
\text { b. } & \neg x \mathrm{P} y \wedge \neg x \mathrm{I} y .
\end{array}
$$

4) Incomparability: From the point of view of DM, there is a high heterogeneity between the alternatives, so he/she cannot set a preference relation between them. It is denoted as $x \mathrm{R} y$, and is expressed in terms of $\sigma(x, y)$ as $x \mathrm{R} y \Rightarrow \sigma(x, y)<0.5 \wedge \sigma(y, x)<0.5$.

5) k-Preference: Represents a doubt between $x \mathrm{P} y$ and $x \mathrm{R} y$, and is denoted as $x \mathrm{~K} y .(x, y) \in \mathrm{K}$ if the following three conditions are true:

$$
\begin{array}{ll}
\text { a. } & 0.5 \leq \sigma(x, y)<\lambda . \\
\text { b. } & \sigma(y, x)<0.5 . \\
\text { c. } & \sigma(x, y)-\sigma(y, x)>\beta / 2
\end{array}
$$

Indifference corresponds to the existence of clear and positive reasons that justify equivalence between the two options. Besides, incomparability represents situations where the DM cannot, or does not want to, express a preference. Strict preference is associated with conditions in which the DM has clear and well-defined reasons justifying the choice of an alternative over the other. However, due to the DM usually has a non-ideal behavior, there exist the weak preference and the $k$-preference. These relations can be considered as "weakened" ways of the strict preference.

The model parameters need to be adjusted according to the specific characteristics of the problem and the DM. This can be done by an interaction between the DM and a decision analyst, utilizing, if necessary, indirect elicitation methods to support this task $([49,50,51])$.

From a set of feasible portfolios $O$, the preferential system defines the following sets:

1) $S(O, x)=\{y \in O \mid y \mathrm{P} x\}$, composed of the solutions that strictly outrank $x$.

2) $N S(O)=\{x \in O \mid S(O, x)=\varnothing\}$, is known as non-strictlyoutranked frontier.

3) $W(O, x)=\{y \in N S(O) \mid y \mathrm{Q} x \vee y \mathrm{~K} x\}$, composed of the non-strictly-outranked solutions that weakly outrank $x$.

4) $N W(O)=\{x \in N S(O) \mid W(O, x)=\varnothing\}$, is known as nonweakly-outranked frontier.

Obviously, solutions that presumed to be the best compromise among a set $O$ of actions must belong to $N S(O)$. However, there may be more than one solution with such feature, so more information is needed to describe the DM's preferences and enhance the optimization process. A solution belonging to $N W(O)$ has a greater potential to be the best compromise that those that do not have this condition.

Besides the weak outranking, the net flow score is another measure used by Fernandez et al. ([10, 30]) to identify the DM's preferences in the non-strictly-outranked frontier. It can be defined as:

$$
F_{n}(x)=\sum_{y \in N S(O) \backslash\{x\}}[\sigma(x, y)-\sigma(y, x)]
$$

Since $F_{n}(x)>F_{n}(y)$ indicates a preference for $x$ over $y$, Fernandez et al. ([10]) define:

1) $F(O, x)=\left\{y \in N S(O) \mid F_{n}(y)>F_{n}(x)\right\}$, as the set of nonstrictly-outranked solutions that surpass in net flow to $x$.

2) $N F(O)=\{x \in N S(O) \mid F(O, x)=\varnothing\}$, is known as net flow non-outranked frontier.

Then, the model proposed in [10] suggests finding the best compromise in $O$ by solving Problem 3:

$$
\min _{x \in O}\{|S(O, x)|,|W(O, x)|,|F(O, x)|\}
$$

with pre-emptive priority favoring $|S(O, x)|$. Fernandez et al. proved in [10] that the best portfolio compatible with the fuzzy outranking relation $\sigma$ should be a $(0,0,0)$ solution to Problem 3 with $O=R_{F}$.

\section{Our proposal}

Fernandez et al. ([10]) solve Problem 3 by using an evolutionary algorithm inspired by NSGA2. This performs well when interdependent projects are not considered. However, if project interaction is addressed, the crossover opera- 
tion could remove convenient synergetic projects from the portfolio. Therefore, we prefer to use a building-oriented metaheuristic approach.

Our algorithm, NO-ACO (Non-Outranked Ant Colony Optimization), is based on the optimization idea proposed in [52] by Dorigo and Gambardella which has been adapted more than once to find a set of Pareto solutions (e.g. [31, 53, 54]). Unlike other multiobjective ant-based optimization methods, NO-ACO incorporates the preference model from [10, 30]. The algorithm performs the optimization process through a set of agents called ants. Each ant in the colony builds a portfolio by selecting a project at a time. The way how to choose each project is called selection rule. When all ants have finished constructing their portfolios, these are evaluated and each ant drops pheromone according to this assessment. Pheromone is a learning kind that allows next generation of ants to acquire knowledge of the structure of the best solutions. To prevent premature convergence, the colony includes a strategic oblivion mechanism, known as evaporation, which reduces the pheromone trail every specified period of time.

In order to improve the intensification, NO-ACO includes a variable neighborhood search for the best solutions. This local search runs once per iteration.

This intensifier scheme is complemented by a diversifier mechanism, in which portfolios that have remained non-strictly-outranked for more than $\gamma$ generations are removed from the solution set. This allows relaxing the selective pressure. This behavior is desirable whether the algorithm has only found out local optima.

The optimization process ends when reaching a predetermined termination criterion, such as a maximum number of iterations, or subsequent recurrence of the best solution. The following sections describe in further detail the elements of NO-ACO algorithm.

\subsection{Pheromone representation}

Pheromone is usually represented by the Greek letter $\tau$ and is modeled in NO-ACO as a two dimensional array of size $N \times N$, where $N$ is the total number of applicant project proposals. The pheromone between two projects $i$ and $j$ is represented as $\tau_{i, j}$, and indicates how good is that both projects receive financial support. Pheromone values are in range $(0,1]$, initializing at the upper limit to prevent premature convergence. The pheromone matrix acts as a reinforcement learning structure reflecting the knowledge gained by ants that formed high-quality portfolios. Pheromone transmits it to ants of the next generation for building better solutions.

\subsection{Selection rule}

Each ant builds its portfolio by selecting one by one the projects, taking into account two factors:

1) Local knowledge: This considers the benefits provided by the project to the portfolio and how much resource it consumes. Local knowledge for a project $i$ is denoted as $\eta_{i}$ and is calculated by the expression:

$$
\eta_{i}=\frac{\frac{1}{c_{i}} \sum_{j=0}^{p} f_{j}(i)}{\max _{k \in X}\left\{\frac{1}{c_{k}} \sum_{j=0}^{p} f_{j}(k)\right\}}
$$

where $c_{i}$ is the cost of project $i, p$ is the number of objectives, $X$ is the applicant project list, and $f_{j}(i)$ the benefits of the project $i$ to the $j$ th objective. Formula 4 promotes the inclusion of projects that have a good balance between intended objectives and requested budget.

2) Global knowledge: This takes into account the experience of previous generations ants, expressed in the pheromone matrix. The global knowledge for the project $i$ to be included in a portfolio $x$ is denoted by $\overline{\tau(x, i)}$ and is defined by the expression:

$$
\overline{\tau(x, i)}=\frac{\sum_{j=0}^{N}\left(x_{j}\right) \tau_{i, j}}{\sum_{j=0}^{N} x_{j}}
$$

where $N$ is the total number of applicant projects, $x_{j}$ is the binary value indicating whether the $j$ th project is included in the portfolio $x$, and $\tau_{i, j}$ is the pheromone for projects $i$ and $j$. The numerator in (5) is the total sum of pheromone between $i$ and each project in the portfolio $x$; and the denominator is the cardinality of $x$. The global knowledge favors the selection of projects that were part of the best portfolios in previous generations. At the first iteration this knowledge has no effect on portfolio formation process.

Both knowledge factors are linearly combined into a single evaluation function:

$$
\Omega(x, i)=w \cdot \eta_{i}+(1-w) \cdot \overline{\tau(x, i)}
$$

where $w$ is a weight parameter between global and local knowledge, and should receive a value between zero and one. Each ant in the colony has a different value for $w$ generated at random. Function $\Omega$ forms the basis of the selection rule.

If $x$ is a partially-constructed portfolio, one or more projects may be included to $x$. From among all project proposals, only those ones that are not part of $x$ and whose inclusion favors the fulfillment of budgetary constraints should be considered. This set is known as candidate project list and is denoted by $X^{\ominus}$. Note that $X^{\square \Theta}$ is a subset of $X$. The choice of what $j \in X^{\Theta}$ will be added is made by using the selection rule: 


$$
j= \begin{cases}\arg \max _{i \in X^{\theta}}\{\Omega(x, i)\} & \text { if } \wp \leq \alpha_{1}, \\ \mathcal{L}_{i \in X^{\theta}}\{\Omega(x, i)\} & \text { if } \alpha_{1}<\wp \leq \alpha_{2}, \\ \ell_{i \in X^{\theta}} & \text { otherwise. }\end{cases}
$$

where $j$ is the next project to be included, $\wp$ is a pseudorandom number between zero and one; $\alpha_{1}$ is a parameter that sets the intensification probability in the algorithm (choosing the project with the greatest value of $\Omega$ ); whilst $\alpha_{2}-\alpha_{1}$ is the probability to trigger a middle state between intensification and diversification (selecting randomly a project $i$ with probability proportional to its assessment $\Omega$ ), this selection scheme is represented by $\mathcal{L}$; in the event that $\wp>\alpha_{2}$, diversification is promoted by means of the function $\ell$ (taking a project uniformly at random).

\subsection{Pheromone laying and evaporation}

At the beginning of the first iteration, the pheromone matrix is initialized to $\tau_{i, j}=1$ for all $(i, j) \in N \times N$. After that, each ant constructs a feasible portfolio. In a colony with $n$ ants, $n$ new solutions are generated at the end of each iteration, and also there is a set of size $m$ with the best portfolios found out in previous iterations. If all alternatives are integrated into a set $O$ whose cardinality is $n+m$, we can identify the non-strictly-outranked front $N S(O)$.

In addition, $N S(O)$ is subdivided into domination fronts similarly to NSGA-II ([32]). The fronts are obtained considering two objectives to minimize: $W(O, x)$ and $F(O, x)$, according to the best-compromise definition given in (3). The set composed by these fronts is denoted by $F=\left\{F_{1}, F_{2}, \ldots, F_{k+1}, \ldots\right\}$, where $F_{1}$ contains the nondominated solutions, $F_{2}$ contains the dominated by only one solution, $F_{3}$ the dominated by two solutions, and so forth. In general, the solutions dominated by $k$ solutions are in $F_{k+1}$. The set $F$ will be used in the pheromone intensification in order to increase the selective pressure towards the best compromise.

Each pair of projects $(i, j)$ for each solution $c \in O$ intensifies the pheromone trail according to the expression:

$$
\tau_{i, j}= \begin{cases}\tau_{i, j}+\Delta \tau_{i, j} & \text { if } c \in N S(O) \\ \tau_{i, j} & \text { otherwise }\end{cases}
$$

If $c$ is a non-strictly-outranked solution, then there is a $k$ such that $c \in F_{k}$. The pheromone increase depends on $k$, and is defined as:

$$
\Delta \tau_{i, j}=\left(\frac{|F|-k+1}{|F|}\right)\left(1-\tau_{i, j}\right) \quad \text { if } c \in F_{k} .
$$

If there are cycles in the strict preference relation, no solution can be identified into $N S(O)$. This may result from a wrong settlement of model parameters; in this case, a closer interaction with the DM will be required for reaching a consistent preference representation. Another reason may be a high heterogeneity in preferences when the DM is a conflicting group.

At the end of each iteration, the entire pheromone matrix is evaporated through a multiplication by a constant factor between zero and one, denoted as $\rho$.

\subsection{Local search}

The algorithm intensification is promoted by a greedy variable-neighborhood local search that is only carried out on non-strictly-outranked solutions. This search explores regions near to the best known solutions by a simple scheme consisting of selecting randomly $v$ projects, and generating all possible combinations of them for each solution in the non-strictly-outranked frontier. Small values for $v$ provoke a too greedy behavior, whereas large values produce intolerable computation times. In our experiments we obtained a good balance between both by using $v=\lceil\ln N\rceil$.

\section{Case study: Optimization of social assistance port- folios}

Consider a DM facing a portfolio problem, with 100 project proposals that attempt to benefit the most precarious social classes. The project quality is measured as the number of beneficiaries for each of nine criteria established previously. Each objective is associated to one of three classes (extreme poverty, lower class and lowermiddle class) and one of three levels of impact (low, medium and high). 
Table 1: Effect of preferences incorporation on the Pareto Ant Colony Optimization algorithm

\begin{tabular}{|c|c|c|c|c|c|c|}
\hline Instance & Algorithm & $\begin{array}{l}\text { Time (se- } \\
\text { conds) }\end{array}$ & $\begin{array}{l}\text { Size of the } \\
\text { solution set }\end{array}$ & $\begin{array}{c}\text { Non-dominated } \\
\text { solutions in } \\
O_{1} \cup O_{2}\end{array}$ & $\begin{array}{c}\text { Solutions belong- } \\
\text { ing to } N S\left(O_{1} \cup\right. \\
\left.O_{2}\right)\end{array}$ & $\begin{array}{c}\text { Obtains the best } \\
\text { compromise in } \\
O_{1} \cup O_{2}\end{array}$ \\
\hline \multirow{2}{*}{1} & P-ACO & 3448.07 & 2006 & 928 & 10 & \\
\hline & $\mathrm{P}-\mathrm{ACO}-\mathrm{P}$ & 536.66 & 15 & 15 & 10 & $\tau$ \\
\hline \multirow{2}{*}{2} & P-ACO & 3470.29 & 2514 & 1295 & 7 & \\
\hline & P-ACO-P & 775.94 & 19 & 19 & 13 & 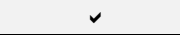 \\
\hline \multirow{2}{*}{3} & P-ACO & 3485.16 & 2456 & 280 & 13 & \\
\hline & $\mathrm{P}-\mathrm{ACO}-\mathrm{P}$ & $1112.49^{-}$ & 34 & 34 & 17 & 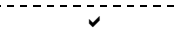 \\
\hline \multirow[b]{2}{*}{4} & P-ACO & 3591.27 & 2587 & 1392 & 10 & $\therefore$ \\
\hline & P-ACO-P & 734.58 & 38 & 37 & 19 &  \\
\hline \multirow[b]{2}{*}{5} & P-ACO & 3525.85 & 2245 & 1165 & 10 & \\
\hline & $\mathrm{P}-\mathrm{ACO}-\mathrm{P}$ & 1035.85 & $21^{-}$ & $21^{-}$ & 15 & $\because$ \\
\hline
\end{tabular}

The total budget to distribute is 250 million dollars. The proposals can be grouped into three types according to their nature, and into two geographic regions according to the impact location. Furthermore, desiring to provide equitable conditions, the DM imposes the following restrictions: 1) the budget allocated to support each project type should vary between $20 \%$ and $60 \%$ of the total budget, and 2) the financial support allocated to each region must be at least $30 \%$ of the total, and no more than $70 \%$.

Also, the DM has identified 20 relevant interactions among projects: four of them are cannibalization phenomena, six correspond to situations of mutually excluding projects, and ten are synergism interactions. There are up to five projects per interaction. Into our algorithm, these relations are modeled as in [5].

Below, we present a range of experiments to verify the validity and advantages of our approach to solve this case study. They give evidence of the benefits of incorporating DM preferences during the optimization process, and thus, they also prove that our approach has good potential in solving real resource-allocation problems.

\subsection{Effect of the DM's preference incorporation}

In order to appraise which is the effect on a multiobjective optimization algorithm by incorporating DM preferences, we implemented the P-ACO algorithm proposed by Doerner et al. in [31].

To the best of our knowledge, P-ACO is the most prominent ant colony algorithm applied to solve project portfolio selection.

We also developed a version of P-ACO including the preferential model described in Section 3. This adaptation was called $\mathrm{P}-\mathrm{ACO}$ with preferences (P-ACO-P). The latter, instead of approximating the Pareto frontier defined by the nine maximizing objectives of the problem, searches the best compromise expressed by (3). It is easy to prove that the set of solutions pursued by $\mathrm{P}-\mathrm{ACO}-\mathrm{P}$ is a subset of P-ACO's.

In order to reflect a credible decision situation, we assign the values suggested by Fernandez et al. in [30] to the preferential model parameters.
Both algorithms were programmed in Java language, using the JDK 1.6 compiler, and NetBeans 6.9.1 as integrated development environment. The experiments were run on a Mac Pro with processor Intel Quad-Core 2.8 $\mathrm{GHz}$ and $3 \mathrm{~GB}$ of RAM. The P-ACO parameter setting was the suggested by Doerner et al. ([31]). The version that incorporates preferences has the same setting values.

Table 1 shows the experimental results on five artificial instances following the case-study features.

As is observed in Table 1, incorporating preferences provides a closer approximation to a privileged region of the Pareto frontier. The version considering preferences provides solutions that dominated the $57 \%$, on average, of solutions from the algorithm original version. There is also a significant run-time reduction (in the test cases, it was $76 \%$ on average). Also, if the model of preferences matches with the DM's preferences, the real best compromise among the set of all portfolios generated is always identified by P-ACO-P. Furthermore, when the DM has to choose one alternative as the final decision, the thousands of portfolios from P-ACO shall make difficult to make a decision. By incorporating preferences, this drawback is very strongly reduced.

\subsection{Analysis of the algorithm performance}

This section is presented with the intention to provide evidence of the performance of our algorithm NO-ACO. The main differences from the P-ACO-P (with preferences) are presented in Table 2 . In order to verify whether the NO-ACO strategies have been properly instantiated, in this section we compare the performance of NO-ACO with $\mathrm{P}-\mathrm{ACO}$ incorporating preferences.

We have deactivating the local search of our algorithm, with the intention of achieving comparison conditions as balanced as possible.

The experimental results are shown in Table 3, where can be observed that, although P-ACO-P finds larger solution sets, most of these are suboptimal solutions with respect to NO-ACO's. So the non-strictly-outranked frontier is better approximated by NO-ACO. 
Table 2: Main differences between P-ACO (with preferences) and NO-ACO algorithms

\begin{tabular}{|c|c|c|}
\hline Algorithm element & The way like P-ACO-P carries out it & The way like NO-ACO carries out it \\
\hline $\begin{array}{l}\text { Pheromone represen- } \\
\text { tation }\end{array}$ & $\begin{array}{l}\text { A two-dimensional matrix with size } N^{\prime} p \text {. Where } \\
N \text { is the number of applicant projects and } p \text { is the total of } \\
\text { criteria. }\end{array}$ & A two-dimensional matrix with size $N^{\prime} N$ \\
\hline Pheromone laying & $\begin{array}{l}\text { The best and the second best solutions for each ob- } \\
\text { jective intensify the pheromone. }\end{array}$ & $\begin{array}{l}\text { The solutions intensify the pheromone according to } \\
\text { dominance fronts. }\end{array}$ \\
\hline $\begin{array}{l}\text { Pheromone evapora- } \\
\text { tion }\end{array}$ & The ants do it during the solution building. & $\begin{array}{l}\text { The entire pheromone matrix is evaporated once per } \\
\text { iteration. }\end{array}$ \\
\hline Lifespan for the ants & $\begin{array}{l}\text { It is randomly generated. Every time an ant adds a } \\
\text { project, the lifespan is decreased by one. }\end{array}$ & $\begin{array}{l}\text { It is equal to budget. Every time an ant adds a project, } \\
\text { the project cost is deducted from the lifespan. }\end{array}$ \\
\hline Local knowledge & It promotes the forming of feasible portfolios. & $\begin{array}{l}\text { It promotes the inclusion of projects with higher ratio } \\
\text { between benefits and cost. }\end{array}$ \\
\hline $\begin{array}{l}\text { Ignoring old solu- } \\
\text { tions of the search } \\
\text { process. }\end{array}$ & It is not considered. & $\begin{array}{l}\text { Non-strictly-outranked solutions with more than } \lambda \text { it- } \\
\text { erations are taken out from the search. }\end{array}$ \\
\hline
\end{tabular}

In addition, for all test instances, our proposal is able to identify the best compromise from both solution sets. Concerning run times, there are no significant differences according to a Student's $t$ test for paired samples, using a confidence level of $90 \%$.

The NO-ACO parameter setting used to obtain the results in Table 3 is: $\alpha_{1}=0.65, \alpha_{2}=0.75, \rho=0.10$, $\gamma=5$, rep $\max =21$, and $i t e r_{\max }=1000$. Moreover, the colony has one hundred ants. This setting was obtained from exploring parameters values with the objective of achieving a good algorithmic performance.

\subsection{Solving problems with high dimensionality}

The tests shown in this section are limited to one hundred projects and nine objectives. These dimensions exceed those addressed by most studies in the scientific literature (e.g. [24, 25, 31, 61]). These dimensions are appropriate for most portfolio problems in the business sector; however, in public organizations, the problem size may be larger. In order to explore the capacity of our algorithm to solve instances with a large size, we generated a set of instances with 500 projects and 16 criteria to optimize.

The interpretation is similar to that described at the beginning of this section: there is a budget to distribute to 250 million dollars, also the DM want to keep balancing conditions and has grouped the projects into two areas and in three regions and imposed budgetary constraints for each one $30-70 \%$ for each area and $20-60 \%$ for each region). In addition, the DM has identified 100 relevant interactions between projects: 20 are cannibalization phenomena, 30 correspond to redundancy among projects and 50 are synergies that generate added value.

Unlike the 100-projects case, in these instances it is not possible to generate an acceptable approximation of the Pareto frontier that can be used as reference for comparison purposes. Even the best multiobjective algorithms are degraded attempting to generate it. This combined with computation times that would be intolerable or an abrupt interruption of the algorithms if they fail to converge towards the frontier.

Among several heuristics frequently used, we chose one based on assigning budgetary resources according to project-ranking information. Here, a project ranking is built by using a cost-benefit ratio; the benefit is modeled by a weighted sum, whose weights are adjusted to reflect the DM's preferences. The project ranking is built following the order given by the cost-benefit ratio.

Once the set of projects has been ranked, the resources may be allocated by following the priorities implicit in the rank order until no resources are left. This ensures, at least, the inclusion of projects that provide more benefit per dollar.

Synergism can be tackled if the inter-projects interactions are modeled as dummy projects that can be ranked. Table 4 concentrates only five of 164 solutions found out by NO-ACO as an approximation to non-strictlyoutranked frontier. Our algorithm converges after 21,625 seconds. The best compromise found (Solution 1) outperforms the ranking-based portfolio, even in Pareto sense.

Table 3: Comparative analysis of the NO-ACO performance

\begin{tabular}{|c|c|c|c|c|c|c|}
\hline Instance & Algorithm & $\begin{array}{l}\text { Time (se- } \\
\text { conds) }\end{array}$ & $\begin{array}{l}\text { Size of the } \\
\text { solution set }\end{array}$ & $\begin{array}{c}\text { Non-dominated } \\
\text { solutions in } \\
O_{1} \cup O_{2} \\
\end{array}$ & $\begin{array}{c}\text { Solutions be- } \\
\text { longing to } \\
N S\left(O_{1} \cup O_{2}\right) \\
\end{array}$ & $\begin{array}{c}\text { Obtains the best } \\
\text { compromise in } \\
O_{1} \cup O_{2}\end{array}$ \\
\hline \multirow{2}{*}{1} & P-ACO-P & 536.66 & 15 & 0 & 0 & \\
\hline & $\mathrm{NO}-\mathrm{ACO}$ & 248.68 & 10 & 10 & 10 & \\
\hline \multirow{2}{*}{2} & P-ACO-P & 775.94 & 19 & 0 & 0. & \\
\hline & NO-ACO & 891.76 & 6 & 6 & 6 & $\checkmark$ \\
\hline \multirow{2}{*}{3} & P-ACO-P & 1112.49 & 34 & 0 & 0. & \\
\hline & $\mathrm{NO}-\mathrm{ACO}$ & 789.09 & 5 & 5 & 5 & $\checkmark$ \\
\hline \multirow{2}{*}{4} & P-ACO-P & 734.58 & 38 & 0 & 0. & \\
\hline & $\mathrm{NO}-\mathrm{ACO}$ & $\overline{6} \overline{6} . \overline{9} 8$ & 7 & 7 & 7 & 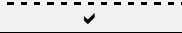 \\
\hline \multirow{2}{*}{5} & P-ACO-P & 1035.85 & 21 & 16 & 9. & \\
\hline & NO-ACO & 456.43 & 10 & 10 & 9 & $\checkmark$ \\
\hline
\end{tabular}

Note: $O_{1}$ and $O_{2}$ are the solution sets generated by P-ACO and P-ACO-P respectively

The best compromise is a $(0,0,0)$ solution to Problem 3 
Table 4: A sample of the non-strictly-outranked frontier generated by NO-ACO compared to the ranking-based solution.

\begin{tabular}{|c|c|c|c|c|c|c|c|c|c|c|c|c|c|c|c|c|c|c|c|c|}
\hline & \multirow{2}{*}{ Portfolio } & \multicolumn{16}{|c|}{ Values of objective functions } & \multicolumn{3}{|c|}{ Number of solutions that outranks it } \\
\hline & & 1 & 2 & 3 & 4 & 5 & 6 & 7 & 8 & 9 & 10 & 11 & 12 & 13 & 14 & 15 & 16 & strictly & weakly & $\begin{array}{l}\text { in net flow } \\
\text { score }\end{array}$ \\
\hline \multirow{5}{*}{$\mid \begin{array}{l}0 \\
\frac{0}{1} \\
0 \\
z \\
z \\
\vdots\end{array}$} & 1 & 10 & & & & & & & & 98 & & 473 & & & 847 & 495 & 597 & 0 & 0 & 0 \\
\hline & 2 & 0 & & & & & & 451 & & & & 457 & כסכ & & 756 & 454 & 545 & 0 & 0 & 1 \\
\hline & 3 & 8 & 730 & 46 & & 99 & 74 & 475 & 56 & 98 & 79 & 46 & 563 & 95 & 767 & 453 & 541 & 0 & 1 & 2 \\
\hline & 4 & 0 & & & 54 & 4 & & 459 & & 992 & & & 547 & & & 447 & 535 & 0 & 2 & 1 \\
\hline & 5 & 96 & & 462 & & 95 & 751 & 456 & 562 & 999 & 809 & 454 & 562 & & 76 & 452 & 546 & 0 & 2 & 1 \\
\hline & $\begin{array}{l}\text { Ranking- } \\
\text { based }\end{array}$ & 96 & 736 & 471 & 558 & 95 & 762 & 453 & 561 & 944 & 768 & 469 & 565 & 97 & 756 & 436 & 540 & 5 & 0 & 5 \\
\hline
\end{tabular}

Another ten instances were generated following the same features. When they were solved by NO-ACO, we observe the same behavior: the ranking-based portfolio was dominated by the best compromise by NO-ACO. This test gives some evidence of the applicability of our approach to solve large-scale real instances.

\section{Conclusions and future work}

We have presented an original proposal to optimize interdependent projects portfolios. This proposal is an adaptation of the well-known Ant Colony Optimization metaheuristic, but incorporating preferences based on the outranking model by Fernandez et al. ([10]).

Our algorithm (NO-ACO) searches for optimal portfolios in synergetic conditions and can handle interactions impacting both objectives and costs. Redundancy is also considered during portfolio formation.

By incorporating preferences, the selective pressure toward a privileged zone of the Pareto frontier is increased. Thus, a zone that matches better the DM's preferences can be identified.

In comparison with other metaheuristic approaches that do not incorporate preferences, NO-ACO achieves a better closeness to the true Pareto front with less computational effort.

Being enriched by preferences, our proposal acquires the ability to solve efficiently portfolio problems with higher dimensions than those reported in scientific literature.

Compared to the popular ranking-based method, NOACO finds out solutions that outperform to the rankingbased portfolio, both in Pareto dominance and strict outranking.

As future work we are going to add the alternative of partial project support. It will also be important to explore the limits of this approach, by finding the top size within instances can be solved with acceptable performance.

\section{References}

[1] Kleinmuntz, D.N. (2011). "Improved methods for resource allocation", in Salo, A., Keisler, J. and Morton, A. (eds.) Portfolio Decision Analysis, Improved methods for resource allocation, Springer, New YorkDordrecht-Heidelberg-London, v-vii.
[2] Salo, A., Keisler, J, Morton, A. (2011). “An invitation to Portfolio Decision Analysis", in Salo, A., Keisler, J., Morton, A. (eds.), Portfolio Decision Analysis, Improved methods for resource allocation, Springer, New York-Dordrecht-Heidelberg-London, 3-27.

[3] Coffin, M.A, Taylor, B.W. (1996). "Multiple criteria R\&D project selection and scheduling using fuzzy sets", Computers \& Operations Research, 23(3): 207220.

[4] Klapka, J., Pinos, P., and Sevcik, V. (2013). "Multicriterial Projects Selection", Handbook of Optimization, Intelligent Systems Reference Library, vol. 38, Springer, 245-261.

[5] Stummer, C. and Heidenberger, K. (2003). "Interactive R\&D Portfolio Analysis with Project Interdependencies and time Profiles of Multiple Objectives". IEEE Transactions on Engineering Management, 50:175-183.

[6] Ringuest, J.L., Graves, S.B., and Case, R.H. (2004). "Mean-Gini analysis in R\&D portfolio selection", $E u$ ropean Journal of Operational Research, 154(1): 157-169.

[7] Carlsson, Ch., Fuller, R., Heikkila, M., Majlender, P. (2007). "A fuzzy approach to R\&D portfolio selection”, International Journal of Approximate Reasoning, 44 (2): 93-105.

[8] Zhao, X., Yang, Y., Wu, G., Yang, J., and Xue, X. (2012). "A dynamic and fuzzy modeling approach for multi-objective R\&D project portfolio selection", Journal of Convergence Information Technology, 7(1): 36-44.

[9] Hallerbach, W., Ning, H., Soppe, A., and Spronk, J., "A framework for managing a portfolio of socially responsible investments", European Journal of Operational Research, 153(2):517-529

[10] Fernandez, E., Lopez, E., Mazcorro, G., Olmedo, R., and Coello, C. (2013). "Application of the NonOutranked Sorting Genetic Algorithm to public project portfolio selection", Information Sciences, 228: 131-149.

[11] Georgia Department of Transportation (2010). Project list and final investment report. Available in http:// www.dot.ga.gov/ localgovernment/ FundingPrograms/ transreferendum/ Pages/ProjectList.aspx (October 4th, 2012).

[12] Georgia Department of Transportation (2012a). Central Savannah River Area, unconstrained project list by county. Available in http:// www.it3.ga.gov/ Doc- 
uments/ UnconstrainedList/ CentralSavannahUnconstrainedlist.pdf (October 4th, 2012).

[13] Georgia Department of Transportation (2012b). Heart of Georgia, Altamaha unconstrained project list by county. Available in http:// www.it3.ga.gov/ Documents/ UnconstrainedList/ HeartofGeorgiaUnconstrainedList-FullSet.pdf (October 4th, 2012).

[14] Georgia Department of Transportation (2012c). River Valley Area, unconstrained project list by county. Available in http:// www.it3.ga.gov/ Documents/ UnconstrainedList/ UnconstrainedList.pdf (October 4th, 2012).

[15] Hwang, C. L., and Masud, A.S. (1979). "Multiple Objective Decision Making. Methods and Applications", Lecture Notes in Economic and Mathematical Systems, vol. 164, Springer Verlag, Berlin.

[16] Ghasemzadeh, F., Archer, N., Iyogun, P., (1999), “A zero-one model for project portfolio selection and scheduling", Journal of the Operational Research Society, 50(7): 745-755.

[17] Amiri, B. (2012). "A multi-objective hybrid optimization algorithm for project selection problem", Journal of Basic and Applied Scientific Research, 2(7): 6995-7002.

[18] Carazo, A. F., Contreras, I., Gómez, T., Pérez, F. (2012). "A project portfolio selection problem in a group decision-making context". Journal of Industrial and Management Optimization; 8 (1); 243-261.

[19] Kremmel, T., Kubalik, J., and Biffl, S. (2011). "Software project portfolio optimization with advanced multi-objective evolutionary algorithm", Applied Soft Computing, 11(1): 1416-1426.

[20] Chen, A. and Chyu, Ch. (2010). "Applying memetic algorithm in multi-objective resource allocation among competing projects", Journal of Software, 5(8): 802-809.

[21] Gaytán, J., and García, J. (2009). "Multicriteria decision on interdependent infrastructure transportation projects using an evolutionary-based framework", Applied Soft Computing 9(2): 512-526.

[22] Ghorbani, S., and Rabbani, M. (2009). "A new multiobjective algorithm for a project selection problem", Advances in Engineering Software, 40(1): 9-14.

[23] Lin, Ch. M., and Gen, M. (2008). "Multicriteria human resource allocation for solving multistage combinatorial optimization problems using multiobjective hybrid genetic algorithm", Expert Systems with Applications, 34(4): 2480-2490.

[24] Doerner, K., Gutjahr, W., Hartl, R., Strauss, C., and Stummer, C. (2006). "Pareto ant colony optimization with ILP preprocessing in multiobjective project portfolio selection". European Journal of Operational Research, 171(3):830 - 841.

[25] Carazo, A. F., Gómez, T., Molina, J., HernándezDíaz, A. G., Guerrero, F. M., and Caballero, R. (2010). "Solving a comprehensive model for multiobjective project portfolio selection". Computers \& Operations Research, 37(4):630-639.
[26] Zitzler E, Laumanns M, Thiele L. SPEA2: Improving the strength Pareto evolutionary algorithm. Technical report No. 103, Computer Engineering and Networks Laboratory (TIK), Swiss Federal Institute of Technology (ETH), Zurich, Switzerland, 2001.

[27] Coello, C. A. C. (1999). "An updated survey of evolutionary multiobjective optimization techniques: State of the art and future trends". In Proceedings of the Congress on Evolutionary Computation, pp. 3-13. IEEE Press.

[28] Wang, Y., and Yang, Y. (2009). "Particle swarm optimization with preference order ranking for multiobjective optimization", Information Sciences, 179(12): 1944-1959.

[29] Coello, C., Van Veldhuizen, D., Lamont, G., (2007). "Evolutionary Algorithms for Solving MultiObjective Problems", Springer, New York.

[30] Fernandez, E. R., Lopez, E., Lopez, F., and Coello, C. A. C. (2011). "Increasing selective pressure towards the best compromise in evolutionary multiobjective optimization: The extended NOSGA method". Information Sciences, 181(1):44- 56.

[31] Doerner, K. F., Gutjahr, W. J., Hartl, R. F., Strauss, C., and Stummer,C. (2004). "Pareto ant colony optimization: A metaheuristic approach to multiobjective portfolio selection". Annals OR, 131(1-4):79-99.

[32] Deb, K. (2001). "Multi-Objective Optimization Using Evolutionary Algorithms". Wiley, first edition.

[33] "Knowles, J. and Corne, D. (2000). M-PAES: A memetic algorithm for multiobjective optimization". In Evolutionary Computation, 2000, pp. 325-332. IEEE Press.

[34] Deb, K., Sundar, J., Bhaskara, U., and Chaudhuri, S. (2006). "Reference point based multiobjective optimization using evolutionary algorithms". International Journal of Computational Intelligence Research, 2(3): 273-286.

[35] S. Bechikh, (2013), “Incorporating Decision Maker's Preference Information in Evolutionary Multiobjective Optimization", Diss. PhD thesis, High Institute of Management of Tunis, University of Tunis, Tunisia.

[36] Deb, K. (1999). "Multi-objective evolutionary algorithms: Introducing bias among Pareto optimal solutions". KanGAL Report 99002, Indian Institute of Technology, Kanpur, India.

[37] Branke, J., and Deb, K. (2004). "Integrating user preferences into evolutionary multi-objective optimization". In Y. Jin (ed.), Knowledge Incorporation in Evolutionary Computation, Springer, Berlin, Heidelberg, 461-478.

[38] Köksalan, M., and Karahan, I. (2010). "An interactive territory defining evolutionary algorithm: iTDEA". IEEE Transactions on Evolutionary Computation, 14(5): 702-722.

[39] Battiti, R., and Passerini, A. (2010). "Brain-computer evolutionary multiobjective optimization: A genetic algorithm adapting to the decision maker". IEEE 
Transactions on Evolutionary Computation, 14(5): 671-687.

[40] Jin, Y. C., and Sendhoff, B. (2002). "Incorporation of fuzzy preferences into evolutionary multiobjective optimization". In Proceedings of the 4th Asia-Pacific conference on Simulated Evolution and Learning, Nanyang, Singapore, 26-30.

[41] Cvetkovic, D., and Parmee, I. C. (2002). "Preferences and their application in evolutionary multiobjective optimization". IEEE Transactions on Evolutionary Computation, 6(1): 42-57.

[42] Allmendinger, R., Li, X., and Branke, J. (2008). "Reference point-based particle swarm optimization using a steady-state approach". In Proceedings of the 7th international conference on Simulated Evolution and Learning (SEAL'08), Springer, Melbourne, Australia, 200-209.

[43] Molina, J., Santana-Quintero, L. V., Hernández-Díaz, A.G., Coello Coello, C. A., and Caballero, R. 2009. "g-dominance: Reference point based dominance for multiobjective metaheuristics". European Journal of operational Research, 197(2): 685-692.

[44] Branke, J., Kaussler, T., and Schmeck, H. (2001). "Guidance in evolutionary multi-objective optimization”. Advances in Engineering Software, 32 (6): 499507.

[45] Wagner, T., and Trautmann, H. (2010). "Integration of preferences in hypervolume-based multiobjective evolutionary algorithms by means of desirability functions". IEEE Transactions on Evolutionary Computation, 14(5): 688-701.

[46] Roy, B. (1996). "Multicriteria Methodology for Decision Aiding. Nonconvex Optimization and its Applications". Springer.

[47] Roy, B. (1990). "The Outranking Approach and the Foundations of ELECTRE methods", in Bana e Costa, C.A. (ed.), Reading in multiple criteria decision aid, Springer-Verlag, Berlin , 155-183.

[48] Brans, J. and Mareschal, B. (2005). "Promethee methods". In Greco, S. (ed.), Multiple Criteria Decision Analysis: State of the Art Surveys, International Series en Operations Research \& Management Science, pp. 163-190. Springer-Verlag.

[49] Jacquet-Lagreze, E. and Siskos, Y. (2001). "Preference disaggregation: 20 years of MCDA experience".

[50] Doumpos, M., Marinakis, Y., Marinaki, M., and Zopounidis, C. (2009). "An evolutionary approach to construction of outranking models for multicriteria classification: The case of the electre tri method". European Journal of Operational Research, 199(2):496505.

[51] Fernandez, E., Navarro, J., Mazcorro, G. (2012). "Evolutionary multi-objective optimization for inferring outranking model's parameters under scarce reference information and effects of reinforced preference", Foundations of Computing and Decision Sciences, 37(3): 163-197.
[52] Dorigo, M. and Gambardella, L. M. (1997). “Ant colony system: A cooperative learning approach to the traveling salesman problem". IEEE Transactions on Evolutionary Computation.

[53] Alaya, I., Solnon, C., y Ghedira, K. (2007). “Ant Colony Optimizationfor Multi-objective Optimization Problems". In 19th IEEE International Conference on Tools with Artificial Intelligence (ICTAI), pp. 450 457.IEEE Computer Society.

[54] Chaharsooghi, S. and Kermani, A. H. M. (2008). “An effective ant colony optimization algorithm (ACO) for multi-objective resource allocation problem (MORAP)". Applied Mathematics and Computation, 200(1):167 - 177.

[55] Liesio, J., Mild, P., Salo, A. (2008). "Robust portfolio modeling with incomplete cost information and project interdependency", European Journal of Operational Research, 190(3): 679-695. 